\title{
Constant scalar curvature Kähler metrics on rational surfaces
}

\section{Jesus Martinez-Garcia}

Dept. of Mathematical Sciences,

University of Essex, United Kingdom

\section{Correspondence}

Jesus Martinez-Garcia, Dept. of Mathematical Sciences, University of Essex, United Kingdom.

Email:jesus.martinez-garcia@essex.ac.uk

\section{In memory of}

Carmen García Busnadiego

Present address: Colchester Campus, Wivenhoe Park, Colchester, Essex, CO4

3SQ, United Kingdom

\section{Funding information}

Simons Foundation, Grant/Award Number: 488631

\begin{abstract}
We consider projective rational strong Calabi dream surfaces: projective smooth rational surfaces which admit a constant scalar curvature Kähler metric for every Kähler class. We show that there are only two such rational surfaces, namely the projective plane and the quadric surface. In particular, we show that all rational surfaces other than those two admit a destabilising slope test configuration for some polarisation, as introduced by Ross and Thomas. We further show that all Hirzebruch surfaces other than the quadric surface and all rational surfaces with Picard rank 3 do not admit a constant scalar curvature Kähler metric in any Kähler class.

\section{KEYWORDS}

automorphism groups, Calabi dream manifolds, constant scalar curvature, K-stability, Kähler metrics, rational surfaces, slope stability

MSC (2020)

14E05, 14E07, 14J26, 14J50, 53C55
\end{abstract}

\section{1 | INTRODUCTION}

In this article all varieties are assumed to be algebraic, projective and defined over $\mathbb{C}$. The problem of determining the existence of constant scalar curvature Kähler metrics (cscK metrics for short) on projective manifolds is a driving force in complex geometry, which goes back to Calabi's seminal work [7, 8]. It has been known for some time that cscK metrics are essentially unique in their Kähler class, when they exist $[5,13,15]$. This problem has long been expected to have an algebraic formulation, due to the Yau-Tian-Donaldson conjecture:

Conjecture 1.1 (Yau-Tian-Donaldson [29]). Let $X$ be a smooth variety, and let $L$ be an ample line bundle on $X$. Then $X$ admits a constant scalar curvature Kähler ( $\csc K)$ metric in $c_{1}(L)$ if and only if the pair $(X, L)$ is $K$-polystable.

It is known in different degrees of generality that K-polystability is a necessary condition for the existence of a cscK metric, with the most general result due to Berman, Darvas and Lu [6] following work of Darvas and Rubinstein [14].

Even if Conjecture 1.1 holds in all generality, testing which particular polarisations are K-polystable is not an easy task. Moreover, very little is known on how K-stability varies in $\operatorname{Pic}(X) \otimes \mathbb{Q}$, although in [19] it was shown that if $\operatorname{Aut}(X)$ is finite, then the set of K-stable polarisations form a (possibly empty) open set. Hence, it is natural to introduce the set of $K$-stable polarisations of a projective manifold $X$ :

$$
\operatorname{Amp}^{K}(X)=\{L \in \operatorname{Amp}(X) \otimes \mathbb{Q} \mid(X, L) \text { is K-polystable }\} .
$$


In this article we consider the following natural question:

Question 1.2. Which manifolds satisfy $\operatorname{Amp}^{K}(X)=\operatorname{Amp}^{\mathbb{Q}}(X)$ ? Or analogously, which manifolds admit a cscK metric for all ample line bundles?

We answer this question for rational surfaces:

Theorem 1.3. Given a rational projective surface $S$, $\operatorname{Amp}^{K}(S)=\operatorname{Amp}^{\mathbb{Q}}(S)$ if and only if $S=\mathbb{P}^{2}$ or $S=\mathbb{P}^{1} \times \mathbb{P}^{1}$.

After a first version of this article appeared online [22], Chen and Cheng introduced the term Calabi dream manifolds to describe those compact manifolds which admit an extremal metric for each Kähler class [12]. Notice that every cscK metric is extremal. Hence the following comes natural:

Definition 1.4. A Kähler manifold is a strong Calabi dream manifold if it admits a cscK metric in each Kähler class.

Naturally all strong Calabi dream manifolds are Calabi dream manifolds. Chen and Cheng give some examples of Calabi dream manifolds, which are all in fact strong Calabi dream manifolds [12], including K3 surfaces and surfaces of general type with no self-intersection curves. On the other hand, there are examples of surfaces of general type with curves of negative self-intersection which are not strong Calabi dream manifolds [25]. The name Calabi dream refers to the initial belief of Calabi that an extremal metric may exist in each Kähler class of all manifolds (see [12, §1] for a short historical account of Calabi's programme). Theorem 1.3 suggests that we can expect few manifolds to be Calabi dream manifolds. Indeed, characterising when such metrics exist (and determining obstructions to existence) is an instigator in the study of extremal and cscK metrics. Notice that since the Kähler cone of $\mathbb{P}^{n}$ is generated by $\operatorname{Pic}\left(\mathbb{P}^{n}\right)$, Theorem 1.3 provides a complete classification of strong Calabi dream rational projective surfaces, answering a strong analogue (for rational surfaces) of a question in $[12, \S 2.4]$ for surfaces of general type:

Corollary 1.5. The only projective rational strong Calabi dream surfaces are $\mathbb{P}^{2}$ and $\mathbb{P}^{1} \times \mathbb{P}^{1}$.

We introduce the natural counterpart of (strong) Calabi dream manifolds:

Definition 1.6. A compact manifold is a totally unstable manifold (a strongly totally unstable manifold, respectively) if it does not admit a cscK metric (an extremal metric, respectively) in any of its Kähler classes.

Our algebraic methods can only give a partial answer to the following question:

Question 1.7. Which projective rational surfaces are totally unstable?

When the connected component of the automorphism group is not reductive, the Matsushima-Lichnérowicz obstruction gives the non-existence of cscK metrics. This is precisely what lies behind of the next result:

Lemma 1.8. Let $\mathbb{F}_{n}$ be the $\mathrm{n}$-th Hirzebruch surface and let $S \rightarrow \mathbb{F}_{n}$ be the blow-up at any point. Then $S$ does not admit a cscK metric in any Kähler class. Moreover $\mathbb{F}_{n}$ does not admit a cscK metric if $n>0$. In particular, if $S$ is a rational surface with $\operatorname{rk}(\operatorname{Pic}(S)) \leqslant 3$ and $S \neq \mathbb{P}^{2}, \mathbb{P}^{1} \times \mathbb{P}^{1}$, then $S$ is a totally unstable manifold. Moreover, $\operatorname{Amp}^{K}(S)=\emptyset$.

The case of Hirzebruch surfaces was already known to experts (see [2, Remark 1]) via the Matsushima-Lichnérowicz obstruction. We give a new proof using the Ross-Thomas notion of destabilising slope test-configurations [26]. On the other hand, our proof of Lemma 1.8 when $\operatorname{rk}(\operatorname{Pic}(S))=3$ relies solely on the Matsushima-Lichnérowicz obstruction and as far as we understand, it was not previously published anywhere. In Section $\$ 2$ we recall these constructions and in Section $\S 3$ we apply them to prove our results after giving a summary of the geometry of Hirzebruch surfaces.

While Lemma 1.8 is not likely to give a complete answer to Question 1.7 for rational surfaces, it is the more general statement we can hope to prove in relation to the rank of the Picard group. Indeed, every del Pezzo surface $S$ with $\operatorname{rk}(\operatorname{Pic}(S)) \geqslant 4$ 
has a Kähler-Einstein metric. Furthermore, the blow-up of $S$ at finitely many points has a constant scalar curvature Kähler metric by a result of Arezzo and Pacard [3]. Nevertheless, the Matsushima-Lichérowicz obstruction seems to be too coarse to detect all totally unstable manifolds. Therefore the following question arises naturally:

Question 1.9. Is there any totally unstable manifold $X$ rational and with reductive $\operatorname{Aut}^{0}(X)$ ?

We expect most manifolds to be at some point in between strongly totally unstable manifolds and strong Calabi dream manifolds, but no explicit descriptions of $\operatorname{Amp}^{K}(S)$ are known beyond some simple cases such as toric varieties and some partial results on smooth del Pezzo surfaces $[9,10]$.

\section{2 | DESTABILISING TEST CONFIGURATIONS}

Definition 2.1. Let $(X, L)$ be a pair formed by a projective manifold and an ample line bundle. Let $\mathbb{G}_{m}=\mathbb{C} \backslash\{0\}$ be the multiplicative group. In this article a test configuration of $(X, L)$ (with exponent $r)$ is a triple $(\mathcal{X}, \mathcal{L}, p)$ consisting of

- a normal projective variety $\mathcal{X}$ with a $\mathbb{G}_{m}$-action,

- a flat $\mathbb{G}_{m}$-equivariant map $p: \mathcal{X} \rightarrow \mathbb{P}^{1}\left(\right.$ where $\mathbb{G}_{m}$ acts naturally on $\mathbb{P}^{1}$ ) such that $p^{-1}(t) \cong X$ for every $t \in \mathbb{P}^{1} \backslash\{0\}$,

- a $\mathbb{G}_{m}$-equivariant $p$-ample line bundle $\mathcal{L} \rightarrow \mathcal{X}$, such that

$$
\left.\mathcal{L}\right|_{p^{-1}(t)} \cong L^{\otimes r}
$$

for every $t \in \mathbb{P}^{1} \backslash\{0\}$, where we identify $p^{-1}(t)$ with $X$.

A test configuration $(\mathcal{X}, \mathcal{L}, p)$ is a product test configuration if $\mathcal{X} \cong X \times \mathbb{P}^{1}$ and $\mathcal{L}=p_{1}^{*}\left(L^{\otimes r}\right)$. A product test configuration is trivial if $\mathbb{G}_{m}$ acts trivially on the left factor of $X \times \mathbb{P}^{1}$.

The original definition of test configuration is somewhat different: the fibration $p$ is customarily defined over $A^{1}$ instead of over $\mathbb{P}^{1}$. However, $\mathrm{Li}$ and $\mathrm{Xu}$ showed that the original definition of test configuration compactifies into the one in Definition 2.1 (see [20] or [10, Section 2] for a succinct description of this equivalence). We will use the intersection formula for the generalised Futaki invariant (sometimes known in the literature as Donaldson-Futaki invariant) appearing in [20, Proposition 6] and $[24,30]$ as the definition. We recall that the slope of the pair $(X, L)$ is

$$
v(L)=\frac{-K_{X} \cdot L^{n-1}}{L^{n}}
$$

The generalised Futaki invariant of the test configuration $(\mathcal{X}, \mathcal{L}, p)$ with exponent $r$ is the number

$$
\mathrm{DF}(\mathcal{X}, \mathcal{L}, p)=\frac{1}{r^{n}}\left(\frac{n}{n+1} \frac{1}{r} \nu(L) \mathcal{L}^{n+1}+\mathcal{L}^{n} \cdot\left(K_{\mathcal{X}}-p^{*}\left(K_{\mathbb{P} 1}\right)\right)\right)
$$

where $n$ is the dimension of the variety $X$. If the test configuration $(\mathcal{X}, \mathcal{L}, p)$ is trivial then $(2.1)$ gives $\operatorname{DF}(\mathcal{X}, \mathcal{L}, p)=0$.

Definition 2.2. The pair $(X, L)$ is $K$-polystable if $\operatorname{DF}(\mathcal{X}, \mathcal{L}, p) \geqslant 0$ for every non-trivial test configuration $(\mathcal{X}, \mathcal{L}, p)$, and $\mathrm{DF}(\mathcal{X}, \mathcal{L}, p)=0$ only if $(\mathcal{X}, \mathcal{L}, p)$ is a product test configuration. The pair $(X, L)$ is $K$-stable if $\mathrm{DF}(\mathcal{X}, \mathcal{L}, p)>0$ for every non-trivial test configuration $(\mathcal{X}, \mathcal{L}, p)$. If $\operatorname{DF}(\mathcal{X}, \mathcal{L}, p) \geqslant 0$ for every test configuration $(\mathcal{X}, \mathcal{L}, p)$, then $(X, L)$ is $K$-semistable.

If the pair $(X, L)$ is not $\mathrm{K}$-semistable, then $\operatorname{DF}(\mathcal{X}, \mathcal{L}, p)<0$ for some test configuration $(\mathcal{X}, \mathcal{L}, p)$ of the pair $(X, L)$. In this case, we say that $(X, L)$ is $K$-unstable, and $(\mathcal{X}, \mathcal{L}, p)$ is a destabilising test configuration.

The pair $(X, L)$ is $\mathrm{K}$-polystable (respectively, $\mathrm{K}$-stable or $\mathrm{K}$-semistable) if and only if the pair $\left(X, L^{\otimes k}\right)$ is $\mathrm{K}$-polystable (respectively, K-stable or K-semistable) for some positive integer $k$. Thus, we can adapt both Definitions 2.1 and 2.2 to the case when $L$ is an ample $\mathbb{Q}$-divisor class on the variety $X$ and assume that $r=1$ in the formula (2.1) for the generalised Futaki invariant. This gives us notions of K-polystability, K-stability, K-semistability and K-unstability for 
varieties polarised by ample $\mathbb{Q}$-divisor classes. In the following we will use $\mathbb{Q}$-divisor classes and $\mathbb{Q}$-line bundles interchangeably.

\section{1 | Automorphism groups and the Matsushima-Lichnérowicz obstruction}

Let us recall the following well-known obstruction to the existence of cscK metrics:

Theorem 2.3 (Matsushima-Lichnérowicz's obstruction [21], cf. [27, §4.2]). If $X$ is a smooth complex projective variety admitting a cscK metric, then the connected identity component of the automorphism group $\operatorname{Aut}^{0}(X)$ is reductive.

Remark 2.4. The classification and study of (linearly) reductive linear algebraic groups can become rather technical. Fortunately, for our purposes it will suffice us with the following well known rules (see [23, Chapter 4] for more details):

(i) If $G$ is defined over $\mathbb{C}, G$ is linearly reductive if and only if the connected identity component $G^{0}$ is reductive.

(ii) If $H \triangleleft G$ is normal and $G$ is linearly reductive, then $G / H$ is linearly reductive.

Lemma 2.5. Let $H$ be a reductive connected complex algebraic linear group. Let $H^{\prime} \triangleleft H$ be a finite normal subgroup of $H$. The group $G=\left(\mathbb{G}_{a}\right)^{n+1} \rtimes\left(H / H^{\prime}\right)$ is not reductive for $n \geqslant 0$.

Proof. Notice that $G$ and $H$ are connected and hence $G=G^{0}, H=H^{0}$ and $G$ is linearly reductive if and only if $G$ is reductive. The group $H / H^{\prime}$ is linearly reductive by Remark 2.4 (i) and (ii). The subgroup $H / H^{\prime}$ is normal in $G$ by assumption. If $G$ was linearly reductive, then $G / H=\left(\mathbb{G}_{a}\right)^{n+1}$ would be linearly reductive (and hence reductive) but this is well known to be false (e.g. see [23, Example 4.42] for a proof for $\mathbb{G}_{a}$ and then apply induction on $n$ using Remark 2.4).

The following well-known result will come useful when applying Theorem 2.3.

Lemma 2.6. Let $S$ be a compact complex projective surface and $\pi: S^{\prime} \rightarrow S$ be the blow up of $S$ at a point p. All the automorphisms in $\operatorname{Aut}^{0}\left(S^{\prime}\right)$ leave the exceptional divisor $E=\pi^{-1}(p)$ invariant. Moreover, via restriction to $S^{\prime} \backslash E$, we have

$$
\operatorname{Aut}^{0}\left(S^{\prime}\right) \cong\left\{\phi \in \operatorname{Aut}^{0}(S) \mid \phi(p)=p\right\}=: \operatorname{Aut}^{0}(S, p) .
$$

Proof. An automorphism $\sigma \in \operatorname{Aut}^{0}\left(S^{\prime}\right)$ must leave the exceptional divisor $E$ invariant and hence, it induces an automorphism $\pi_{*} \circ \sigma \in \operatorname{Aut}^{0}(S, p)$ fixing $p$. Conversely, let $\phi: S \rightarrow S$ be an automorphism fixing $p$. The inverse image in $S^{\prime}$ via $\pi$ of both $p$ and $\phi(p)$ is a Cartier divisor. Hence, the universal property of the blow-ups [18, Corollary 7.15] induces a unique automorphism $\widetilde{\phi}: S^{\prime} \rightarrow S^{\prime}$ such that $\pi \circ \phi=\widetilde{\phi} \circ \pi$ and in particular $E$ is $\widetilde{\phi}$-invariant.

\section{2 | Slope stability}

Let us recall the construction of slope test configurations, introduced by Ross and Thomas [26] (also known in the literature as deformation to the normal cone). Our notation follows our previous work with Cheltsov [10]. We have simplified the hypothesis to the needs of the problem. See [10] or [26] for a more general treatment.

Let $S$ be a smooth surface, $L$ be an ample $\mathbb{Q}$-divisor class of $S$ and $Z$ be a smooth irreducible divisor in $S$.

Definition 2.7. The Seshadri constant of $L$ at $Z$ is the real number

$$
\operatorname{Sesh}(S, L, Z)=\sup \{\lambda: L-Z \text { is nef }\}
$$

Let $\pi_{Z}: \mathcal{X} \rightarrow S \times \mathbb{P}^{1}$ be the blow-up of $S \times \mathbb{P}^{1}$ along $Z \times\{0\}$ with exceptional divisor $E_{Z}$. By a slight abuse of notation we identify $Z \subset S$ with $Z \times\{0\} \in S \times \mathbb{P}^{1}$. Let $p_{\mathbb{P}^{1}}: S \times \mathbb{P}^{1} \rightarrow \mathbb{P}^{1}$ and $p_{S}: S \times \mathbb{P}^{1} \rightarrow S$ be the natural projections. Let 
$p=p_{\mathbb{P} 1} \circ \pi_{Z}$ and define the $\mathbb{Q}$-divisor

$$
\mathcal{L}_{\lambda}:=\left(p_{S} \circ \pi_{Z}\right)^{*} L-\lambda E_{Z}
$$

We call $\left(\mathcal{X}, \mathcal{L}_{\lambda}\right)$ the slope test configuration of $(S, L)$ centred at $Z$.

Lemma 2.8 [26], cf. [11, Lemma 2.2], [10, Lemma 3.1]. The $\mathbb{Q}$-divisor class $\mathcal{L}_{\lambda}$ is p-ample for all rational $0<\lambda<$ $\operatorname{Sesh}(S, L, Z)$. Moreover its generalised Futaki invariant satisfies

$$
\mathrm{DF}\left(\mathcal{X}, \mathcal{L}_{\lambda}\right)=\frac{2}{3} \nu(L)\left[-3 \lambda^{2} L \cdot Z+\lambda^{3} Z^{2}\right]+\lambda^{2}(2-2 g(Z))+2 \lambda L \cdot Z,
$$

where $g(Z)$ is the genus of $Z$.

The advantage of the Ross-Thomas construction of test configurations is that it allows us to extend destabilising testconfigurations of a pair $(S, L)$ to destabilising test configurations of pairs $\left(S^{\prime}, L^{\prime}\right)$ where $S^{\prime} \rightarrow S$ is any composition of blow-downs of (-1)-curves not supported on the curve $Z \subset S$ and $L^{\prime}$ is some polarisation. This is the content of [26, Corollary 5.29]. We give a more detailed proof of the result for the convenience of the reader, since this is our main tool to prove Theorem 1.3 and we will need a precise statement.

Suppose that $(S, L)$ is destabilised by a slope test configuration $\left(\mathcal{X}, \mathcal{L}_{\lambda}\right)$ centred at a smooth irreducible curve $Z \subset S$ and let $p \in S \backslash Z$ be a point. Let $g: S^{\prime} \rightarrow S$ be the blow-up of $p$ and $G$ be its exceptional curve. Denote by $Z^{\prime}$ the proper transform of $Z$ in $S^{\prime}$. The morphism $g$ induces a blow-up $h: S^{\prime} \times \mathbb{P}^{1} \rightarrow S \times \mathbb{P}^{1}$ of $p \times \mathbb{P}^{1} \subset S \times \mathbb{P}^{1}$. There exists a commutative diagram

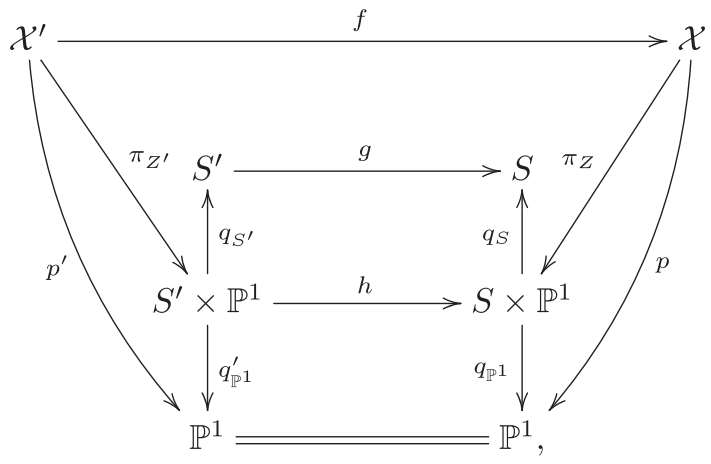

where $q_{S}, q_{S^{\prime}}, q_{\mathbb{P} 1}, q_{\mathbb{P} 1}^{\prime}$ are the obvious projections, $\pi_{Z^{\prime}}: \mathcal{X}^{\prime} \rightarrow S^{\prime} \times \mathbb{P}^{1}$ is the blow-up of $Z^{\prime} \subset S^{\prime} \times\{0\}$ and $f: \mathcal{X}^{\prime} \rightarrow \mathcal{X}$ is the contraction of $G \times \mathbb{P}^{1} \subset \mathcal{X}^{\prime}$. We denote the exceptional divisor of $\pi_{Z^{\prime}}$ by $E_{Z^{\prime}}$.

We may choose some sufficiently small positive rational number $\varepsilon>0$ so that $L_{\varepsilon}^{\prime}=g^{*}(L)-\varepsilon G$ is ample. By Lemma 2.8, we may choose a positive rational number $\lambda=\lambda(\varepsilon)$ such that $0<\lambda<\operatorname{Sesh}\left(S^{\prime}, L_{\varepsilon}^{\prime}, Z^{\prime}\right)$ and then $\mathcal{L}_{\lambda}^{\prime}=\left(p_{S^{\prime}} \circ \pi_{Z^{\prime}}\right)^{*}\left(L^{\prime}\right)-$ $\lambda E_{Z^{\prime}}$ is $p^{\prime}$-ample where $p^{\prime}=q_{\mathbb{P} 1}^{\prime} \circ \pi_{Z^{\prime}},\left(\mathcal{X}^{\prime}, \mathcal{L}_{\lambda}^{\prime}, p^{\prime}\right)$ is a test configuration of $\left(S^{\prime}, L_{\epsilon}^{\prime}\right)$ (in fact its slope test configuration centred at $Z^{\prime}$ ) and

$$
\operatorname{DF}\left(\mathcal{X}^{\prime}, \mathcal{L}_{\lambda}^{\prime}, p^{\prime}\right)=\frac{2}{3} \nu\left(L_{\varepsilon}^{\prime}\right)\left(\lambda^{3} Z^{2}-3 \lambda^{2} L \cdot Z\right)+\lambda^{2}(2-2 g(Z))+2 \lambda L \cdot Z,
$$

where we use the fact that $p \notin Z$. The latter also implies that $\lim _{\varepsilon \rightarrow 0^{+}} \operatorname{Sesh}\left(S^{\prime}, L_{\varepsilon}^{\prime}, Z^{\prime}\right)=\operatorname{Sesh}(S, L, Z)$ and $\nu\left(L_{\varepsilon}^{\prime}\right)=$ $\frac{-K_{S^{\prime}} \cdot L_{\varepsilon}^{\prime}}{L_{\varepsilon}^{\prime} \cdot L_{\varepsilon}^{\prime}}=\frac{-K_{S} \cdot L-\varepsilon}{L^{2}-\varepsilon^{2}}$ so that $\lim _{\varepsilon \rightarrow 0^{+}} \nu\left(L_{\varepsilon}^{\prime}\right)=\nu(L)$. As a result $\lim _{\varepsilon \rightarrow 0^{+}} \operatorname{DF}\left(\mathcal{X}^{\prime}, \mathcal{L}_{\lambda}^{\prime}, p^{\prime}\right)=\operatorname{DF}\left(\mathcal{X}, \mathcal{L}_{\lambda}, p\right)$. In summary, since for fixed $\lambda$ the generalised Futaki invariant $\operatorname{DF}\left(\mathcal{X}^{\prime}, \mathcal{L}_{\lambda}^{\prime}, p^{\prime}\right)$ is a quotient of polynomials on $\epsilon$ (and hence it is continuous) we have proved:

Lemma 2.9 [26, Corollary 5.29]. Suppose that $0<\lambda<\operatorname{Sesh}(S, L, Z)$ and $\operatorname{DF}\left(\mathcal{X}, \mathcal{L}_{\lambda}, p\right)<0$. Then $\lambda<\operatorname{Sesh}\left(S^{\prime}, L_{\varepsilon}^{\prime}, Z^{\prime}\right)$ and $\operatorname{DF}\left(\mathcal{X}^{\prime}, \mathcal{L}_{\lambda}^{\prime}, p^{\prime}\right)<0$ for sufficiently small $\varepsilon>0$. 
We conclude this section by posing the following conjecture:

Conjecture 2.10. Let $(X, L)$ be a pair destabilised by a test configuration $(\mathcal{X}, \mathcal{L})$ and let $\pi: X^{\prime} \rightarrow X$ be a projective birational morphism. There is an ample line bundle $L^{\prime}$ of $X^{\prime}$, a destabilizing test configuration $\left(\mathcal{X}^{\prime}, \mathcal{L}^{\prime}\right)$ of $\left(X^{\prime}, L^{\prime}\right)$, a $K$ unstable pair $\left(X^{\prime \prime}, L^{\prime \prime}\right)$ destabilised by a test configuration $\left(\mathcal{X}^{\prime \prime}, \mathcal{L}^{\prime \prime}\right)$, a birational map $\phi: X \rightarrow X^{\prime \prime}$, a birational morphism $\psi: X^{\prime} \rightarrow X^{\prime \prime}$ satisfying $\psi=\varnothing \circ \pi$ and $\psi\left(L^{\prime}\right)=L^{\prime \prime}$, and morphisms $f: \mathcal{X}^{\prime} \rightarrow \mathcal{X}^{\prime \prime}, g: \mathcal{X}^{\prime} \rightarrow \mathcal{X}$ such that $\mathcal{L}^{\prime \prime}=f_{*}\left(\mathcal{L}^{\prime}\right)$, $\mathcal{L}^{\prime \prime}=g_{*}(\mathcal{L}),\left.f\right|_{F}=\psi$ and $\left.g\right|_{F}=\phi$, where $F$ is the general fibre of $\mathcal{X}^{\prime}$.

There is some additional evidence to support Conjecture 2.10 beyond Lemma 2.9. For instance, it follows from [10, Corollary 3.9] that Conjecture 2.10 holds for the flop slope test configurations introduced by Cheltsov and Rubinstein in [11] when $\pi$ is not supported on the slope curve.

\section{$3 \mid$ PROOFS}

\section{1 | Hirzebruch surfaces}

We recall the basic geometry, positivity, equations and intersection theory of Hirzebruch surfaces. See [4, Chapter IV] and $\left[18\right.$, Chapter V.2] for the details. Denote by $\mathbb{F}_{n}:=\mathbb{P}\left(\mathcal{O}_{\mathbb{P}^{1}} \otimes \mathcal{O}_{\mathbb{P}^{1}}(n)\right) \rightarrow \mathbb{P}^{1}$ the unique $n$-th Hirzebruch surface, where $n \in \mathbb{Z}_{\geqslant 0}$. This is the unique rational ruled surface whose Picard group is isomorphic to $\mathbb{Z} \oplus \mathbb{Z}$ and which contains a unique smooth rational curve of self-intersection $-n$, which is unique if $n>0$. We denote this curve by $Z_{n}$. We denote by $F$ the class of a fibre of the natural projection $\mathbb{F}_{n} \rightarrow \mathbb{P}^{1}$. We have

$$
Z_{n} \cdot F=1, \quad Z_{n}^{2}=-n, \quad F^{2}=0 .
$$

The Mori cone of effective curves $\overline{\mathrm{NE}}\left(\mathbb{F}_{n}\right)$ is two dimensional and generated by $F$ and $Z_{n}$. In the special case where $n=0$, we have $\mathbb{F}_{0} \cong \mathbb{P}^{1} \times \mathbb{P}^{1}$ and the classes $F$ and $Z_{0}$ are the class of a fibre of each of the two different natural projections to $\mathbb{P}^{1}$. Moreover, we have

$$
-K_{\mathbb{F}_{n}} \sim 2 Z_{n}+(n+2) F, \quad-K_{\mathbb{F}_{n}} \cdot F=2, \quad-K_{\mathbb{F}_{n}} \cdot Z_{n}=2-n
$$

The Nef cone $\overline{\operatorname{Nef}}\left(\mathbb{F}_{n}\right)$ is generated by $F$ and $a Z_{n}+(n a) F$, i.e. $C \sim_{\mathbb{Q}} a Z_{n}+b F$ is ample if and only if $a>0$ and $b>n a$.

We can also work with $\mathbb{F}_{n}$ using coordinates. Indeed, its loci is given by

$$
\mathbb{F}_{n}=\left\{([x: y: z],[u: v]) \in \mathbb{P}^{2} \times \mathbb{P}^{1} \mid y v^{n}=z u^{n}\right\} .
$$

In this coordinates the curve $Z_{n}$ is given by

$$
Z_{n}=\left\{([1: 0: 0],[u: v]) \mid(u: v) \in \mathbb{P}^{1}\right\} \subset \mathbb{F}_{n},
$$

and the map $\mathbb{F}_{n} \rightarrow \mathbb{P}^{1}$ is given by projection on the second factor.

Given any rational surface $S \neq \mathbb{P}^{2}$, there is a morphism $S \rightarrow \mathbb{F}_{n}$ for some $n \geqslant 0$, which factors as the contraction of $k \geqslant 0$ $(-1)$-curves. In addition, there is a morphism $\mathbb{F}_{1} \rightarrow \mathbb{P}^{2}$ contracting $Z_{1}$. There is a commutative diagram

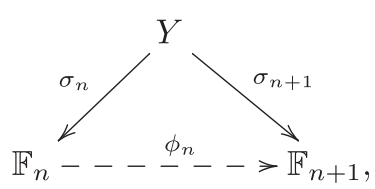

where $\sigma_{n}$ is the blow-up of the point $p_{n}=([1: 0: 0],[1: 0]) \in \mathbb{F}_{n}$ and $\sigma_{n+1}$ is the contraction of the proper transform in $Y$ of the fibre of $\mathbb{F}_{n} \rightarrow \mathbb{P}^{1}$ passing through $p_{n}$. Conversely, up to isomorphism, $\sigma_{n+1}$ is the blow-up of $q_{n+1}=$ $([0: 0: 1],[0: 1]) \in \mathbb{F}_{n+1}$ and $\sigma_{n}$ is the contraction of the proper transform in $Y$ of the fibre of $\mathbb{F}_{n+1} \rightarrow \mathbb{P}^{1}$ passing through $q_{n+1}$. 
We can identify the additive group with the group of homogeneous polynomials of degree $n$ in variables $z_{0}, z_{1}$, i.e. $\left(\mathbb{G}_{a}\right)^{n+1} \cong \mathbb{C}^{n+1} \cong \mathbb{C}\left[z_{0}, z_{1}\right]_{n}$ under this identification. We define the group homomorphism $\phi: \operatorname{GL}(2, \mathbb{C}) \rightarrow \operatorname{Aut}\left(\mathbb{C}^{n+1}\right)$ given by

$$
\phi(M)\left(p\left(z_{0}, z_{1}\right)\right)=p\left(M \cdot\left(z_{0}, z_{1}\right)^{t}\right) .
$$

Using $\phi$, we define the semi-direct product $G_{n}:=\left(\mathbb{G}_{a}\right)^{n+1} \rtimes \mathrm{GL}(2, \mathbb{C})$ with product rule

$$
(p, M) \cdot(q, N)=\left(q+p\left(N \cdot\left(z_{0}, z_{1}\right)^{t}\right), M \cdot N\right) .
$$

Let $n \geqslant 1$. The group $G_{n}$ acts on $\mathbb{F}_{n}$. Indeed, the element

$$
\left(a_{0} z_{0}^{n}+a_{1} z_{1} z_{0}^{n-1}+\cdots+a_{n} z_{1}^{n},\left(\begin{array}{ll}
a & b \\
c & d
\end{array}\right)\right)
$$

acts on the point $([x: y: z],[u: v]) \in \mathbb{F}_{n}$ by sending it to

$$
\left\{\begin{array}{l}
\left(\left[x u^{n}+y\left(a_{0} u^{n}+\cdots+a_{n} v^{n}\right): y(a u+b v)^{n}: y(c u+d v)^{n}\right],[a u+b v: c u+d v]\right), \text { if } u \neq 0, \\
\left(\left[x v^{n}+z\left(a_{0} u^{n}+\cdots+a_{n} v^{n}\right): z(a u+b v)^{n}: z(c u+d v)^{n}\right],[a u+b v: c u+d v]\right), \text { if } v \neq 0 .
\end{array}\right.
$$

Let $\mu_{n} \triangleleft \mathrm{GL}(2, \mathbb{C})$ be the finite subgroup consisting of the diagonal matrices $A=\lambda I$ where $\lambda$ is an $n$-th root of unity. We have an exact sequence of group homomorphisms

$$
1 \longrightarrow \mu_{n} \longrightarrow G_{n} \longrightarrow\left(\mathbb{G}_{a}\right)^{n+1} \rtimes\left(\mathrm{GL}(2, \mathbb{C}) / \mu_{n}\right) \rightarrow 1,
$$

and it is easy to check that $\mu_{n}$ is the kernel of the action $G_{n}$ on $\mathbb{F}_{n}$. Hence $\left(\mathbb{G}_{a}\right)^{n+1} \rtimes\left(\operatorname{GL}(2, \mathbb{C}) / \mu_{n}\right) \subseteq$ Aut $\left(\mathbb{F}_{n}\right)$. While the above description is not entirely necessary to prove the following lemma, it will come useful when studying the automorphism groups in the blow-ups of $\mathbb{F}_{n}$.

Lemma 3.1. Let $S=\mathbb{F}_{n}$ for $n \geqslant 1$. Then $\operatorname{Aut}(S)=\operatorname{Aut}^{0}(S) \cong\left(\mathbb{G}_{a}\right)^{n+1} \rtimes\left(\mathrm{GL}(2, \mathbb{C}) / \mu_{n}\right)$. In particular, $\operatorname{Aut}^{0}(S)$ is not reductive and $S$ does not admit a cscK metric in any Kähler class.

Proof. The contraction of $Z_{n}$ induces a morphism $\pi: \mathbb{F}_{n} \rightarrow S^{\prime}=\mathbb{P}(1,1, n)$. Suppose $n \geqslant 2$. Then $p=\pi\left(Z_{n}\right)=[0: 0: 1]$ is the unique singular point of $S^{\prime}$ and $p$ must be fixed by $\operatorname{Aut}\left(S^{\prime}\right)$. The group $\operatorname{Aut}(S)$ acts transitively on the fibres $\mathbb{F}_{n} \rightarrow \mathbb{P}^{1}$ and it fixes $Z_{n}$, since it is the unique curve in $S$ of negative self-intersection. Hence, by Lemma 2.6 $\operatorname{Aut}(S) \cong \operatorname{Aut}\left(S^{\prime}\right)$. Any $\phi \in \operatorname{Aut}\left(S^{\prime}\right)$ can be given by

$$
\left[t_{0}: t_{1}: t_{2}\right] \mapsto\left[a t_{0}+b t_{1}: c t_{0}+d t_{1}: e t_{2}+p\left(t_{0}: t_{1}\right)\right]
$$

where $p$ is a homogeneous polynomial of degree $n$. Observe that $\left(\mathbb{G}_{a}\right)^{n+1} \triangleleft \operatorname{Aut}\left(S^{\prime}\right)$ is identified with the subgroup of automorphisms given by

$$
\left[t_{0}: t_{1}: t_{2}\right] \mapsto\left[t_{0}: t_{1}: t_{2}+p\left(t_{0}: t_{1}\right)\right]
$$

and $\operatorname{Aut}\left(S^{\prime}\right) /\left(\mathbb{G}_{a}\right)^{n+1} \cong H / \mathbb{G}_{m}$, where $H$ is given by automorphisms of type

$$
\left[t_{0}: t_{1}: t_{2}\right] \mapsto\left[a t_{0}+b t_{1}: c t_{0}+d t_{1}: e t_{2}\right]
$$


and the subgroup $\mathbb{G}_{m}$ consists of diagonal automorphisms $\left[t_{0}: t_{1}: t_{2}\right] \mapsto\left[a t_{0}: a t_{1}: a t_{2}\right]$. Hence $H / \mathbb{G}_{m} \cong \mathrm{GL}(2, \mathbb{C}) / \mu_{m}$ and

$$
\operatorname{Aut}(S) \cong \operatorname{Aut}\left(S^{\prime}\right) \cong\left(\mathbb{G}_{a}\right)^{n+1} \rtimes\left(\mathrm{GL}(2, \mathbb{C}) / \mu_{n}\right) \cong \operatorname{Aut}^{0}(S)
$$

since the group is connected. Now suppose $\pi: S=\mathbb{F}_{n} \rightarrow S^{\prime}=\mathbb{P}^{2}$ is the blow-up of $p=[0: 0: 1] \in \mathbb{P}^{2}$. By Lemma 2.6,

$$
\operatorname{Aut}\left(\mathbb{F}_{1}\right) \cong \operatorname{Aut}\left(\mathbb{P}^{2}, p\right)=\left\{\left(\begin{array}{lll}
1 & a_{0} & a_{1} \\
0 & a & b \\
0 & c & d
\end{array}\right) \in \mathrm{GL}(3, \mathbb{C})\right\} \text {. }
$$

We have a group isomorphism $\operatorname{Aut}\left(\mathbb{F}_{1}\right) \rightarrow\left(\mathbb{G}_{a}\right)^{2} \rtimes \mathrm{GL}(2, \mathbb{C})$, given by

$$
\left(\begin{array}{ccc}
1 & a_{0} & a_{1} \\
0 & a & b \\
0 & c & d
\end{array}\right) \mapsto\left(a z_{0}+a_{1} z_{1},\left(\begin{array}{ll}
a & b \\
c & d
\end{array}\right)\right)
$$

Non-reductivity of $\operatorname{Aut}\left(\mathbb{F}_{1}\right)$ follows from Lemma 2.5 and the fact that $\mathrm{GL}(2, \mathbb{C})$ is linearly reductive [23, Corollary 4.44].

The last lemma and the following one give a complete answer to the existence of cscK metrics on Hirzebruch surfaces.

Lemma 3.2. Let $S \cong \mathbb{P}^{2}$ or $S \cong \mathbb{P}^{1} \times \mathbb{P}^{1}$. Then $S$ admits a cscK metric in any Kähler class. In particular $(S, L)$ is $K$-polystable for all $\mathbb{Q}$-ample line bundles $L$.

Proof. The Picard group of $\mathbb{P}^{n}$ has $\operatorname{rk}\left(\operatorname{Pic}\left(\mathbb{P}^{n}\right)\right)=1$ for all $n \geqslant 1$ and the Kähler cone is one-dimensional. Therefore any ample line bundle $L=\mathcal{O}_{\mathbb{P} n}(a H)$ for some $a \in \mathbb{N}$, where $H$ is the class of a hyperplane. The Fubini-Study metric $g_{F S}$ on $\mathbb{P}^{n}$ is a Kähler-Einstein metric and therefore a cscK metric in $c_{1}(3 H)$. Hence, $\frac{a}{3} g_{F S}$ is a cscK metric in $c_{1}(L)$. In particular this applies to $\mathbb{P}^{2}$. If $L \cong p_{1}^{*} \mathcal{O}_{\mathbb{P} 1}(a H) \otimes p_{2}^{*} \mathcal{O}_{\mathbb{P} 1}(b H)$ is ample and $g_{a}, g_{b}$ are cscK metrics in $c_{1}\left(\mathcal{O}_{\mathbb{P} 1}(a H)\right)$ and $c_{1}\left(\mathcal{O}_{\mathbb{P} 1}(b H)\right)$, then $g_{a}+g_{b}$ is a $\operatorname{cscK}$ metric in $c_{1}\left(\mathcal{O}_{\mathbb{P} 1 \times \mathbb{P} 1}(L)\right)$. Since an $L$-polarised surface $S$ admitting a cscK metric is K-polystable [6, $14]$ and K-polystability is invariant under scaling of $L$, the lemma follows.

Of course, the above lemma is well known and it can be proved in many less direct ways. For instance, since they are toric surfaces, one can use Donaldson's theory $[16,17]$. However, we include the above proof since it is one of the few instances where one could give an explicit description of the metric.

\section{2 | Proof of Theorem 1.3}

While the previous section completes the classification for Hirzebruch surfaces, it does not say anything of an arbitrary rational surface. For this reason we take the following alternative take to Lemma 3.1 via destabilising test configurations:

Lemma 3.3. The pair $\left(\mathbb{F}_{n}, L\right)$, where $n \geqslant 1$ and L is any ample $\mathbb{Q}$-divisor is destabilised by the slope test configuration $\left(\mathcal{X}, \mathcal{L}_{\lambda}\right)$ of $\left(\mathbb{F}_{n}, L\right)$ centred at $Z_{n}$.

Proof. Let $L=a Z_{n}+b F$ be an ample $\mathbb{Q}$-divisor class of $S=\mathbb{F}_{n}$. In particular $a>0$ and $b>n a>0$ and

$$
\nu(L)=\frac{-K_{S} \cdot L}{L^{2}}=\frac{(2-n) a+2 b}{2 a b-a^{2} n} .
$$


Let $Z=Z_{n}$. Then we have

$$
L \cdot Z=b-n a, \quad Z^{2}=-n, \quad L-\lambda Z \equiv(a-\lambda) Z+b F,
$$

and $L-\lambda Z$ is ample if and only if $a>\lambda>\frac{n a-b}{n}$, $\operatorname{sosesh}(X, L, Z)=a$.

Let $\left(\mathcal{X}, \mathcal{L}_{\lambda}\right)$ be the slope test configuration of $(X, L)$ centred at $Z$. Substituting with $L, Z$ and $\lambda=\operatorname{Sesh}(X, L, Z)=a$ in Lemma 2.8 we obtain

$$
\begin{aligned}
\operatorname{DF}\left(\mathcal{X}, \mathcal{L}_{a}\right) & =\frac{2}{3} \cdot \frac{(2-n) a+2 b}{2 a b-n a^{2}}\left(-3 a^{2}(b-n a)-a^{3} n\right)+2 a^{2}+2 a(b-n a) \\
& =\frac{2 a}{3} \cdot \frac{-6 a b+4 a^{2} n+7 n a b-2 n^{2} a^{2}-6 b^{2}}{2 b-n a}+2 a \frac{(a+b-n a) \cdot(6 b-3 n a)}{3(2 b-n a)} \\
& =\frac{2 a}{3} \cdot \frac{-6 a b+4 a^{2} n+7 n a b-2 n^{2} a^{2}-6 b^{2}}{2 b-n a}+2 a \frac{6 a b+6 b^{2}-9 n a b-3 n a^{2}+3 n^{2} a^{2}}{3(2 b-n a)} \\
& =\frac{2 a^{2} n}{3} \cdot \frac{(a-2 b+n a)}{2 b-n a} \\
& <\frac{2 a^{2} n}{3} \cdot \frac{(a-n a)}{2 b-n a} \\
& =\frac{2 a^{3} n}{3} \cdot \frac{(1-n)}{2 b-n a} \leqslant 0
\end{aligned}
$$

as $b>n a>0$ and $n \geqslant 1$. Hence $\operatorname{DF}\left(\mathcal{X}, \mathcal{L}_{a}\right)<0$ for all $n \geqslant 1$. Since $\operatorname{DF}\left(\mathcal{X}, \mathcal{L}_{\lambda}\right)$ is a continuous function on $\lambda$, for some $0<\lambda<\operatorname{Sesh}(S, L, Z)=a$, we have $\operatorname{DF}\left(\mathcal{X}, \mathcal{L}_{\lambda}\right)<0$ and $(S, L)$ is K-unstable.

Remark 3.4. After circulating a version of this article, D. Calderbank let the author know that in [1] the same strategy is followed (namely the construction of a destabilising slope test configuration) to show the obstruction to the existence of extremal metrics on certain ruled surfaces, generalising a technique of Székelyhidi [28]. Their construction tackles the somehow more general case of projectivisation of vector bundles. By contrast, we only use the classification of rational surfaces to construct our destabilising test configuration over any rational surface (other than $\mathbb{P}^{1} \times \mathbb{P}^{1}$ and $\mathbb{P}^{2}$ ). Note that all rational surfaces are blow-ups of Hirzebruch surafaces, which in turn are projectivisations of rank 2 vector bundles over $\mathbb{P}^{1}$ as studied in [1]. We do not rely on the bundle structure, but on the blow-up for the construction of the test configuration.

Proof of Theorem 1.3. Suppose $S \neq \mathbb{P}^{2}$ and $S \neq \mathbb{P}^{1} \times \mathbb{P}^{1}$. We need to find an ample $\mathbb{Q}$-line bundle $L$ such that $(S, L)$ is K-unstable. We will proceed by induction on the $\operatorname{rank} k=\operatorname{rk}(\operatorname{Pic}(S)) \geqslant 2$. By the classification of rational surfaces we have that there is a morphism $f: S=S_{k} \rightarrow S_{k-1} \rightarrow \cdots \rightarrow S_{2}=: \bar{S}=\mathbb{F}_{n}$ which is a composition of blow-ups $S_{i} \rightarrow S_{i-1}$ where $\operatorname{rk}\left(\operatorname{Pic}\left(S_{i}\right)\right)=i$ (or equivalently a composition of contractions of $(-1)$-curves). We may assume that $n \geqslant 1$. Indeed, if $n=0$, then the last blow-up is $f_{2}: S_{3} \rightarrow S_{2}=\mathbb{P}^{1} \times \mathbb{P}^{1}$ where $S_{3}$ is the smooth del Pezzo surface of degree 7 which has three (-1)-curves $E_{1}, E_{2}, E_{3}$ where $E_{2} \cdot E_{3}=E_{2} \cdot E_{1}=1$ and $E_{1} \cdot E_{3}=0$ and $f_{2}$ is the contraction of $E_{2}$. However, we may replace $f_{2}$ by the contraction of $E_{1}$ and then $f_{2}: S_{3} \rightarrow \mathbb{F}_{1}$.

If $k=2$, then $f$ is an isomorphism and the proof follows from Lemma 3.3. Suppose $k=3$ and $f: S=S_{3} \rightarrow \mathbb{F}_{n}$ is the blow-up at a point $p$. If $p \notin Z_{n} \subset \mathbb{F}_{n}$, then the proof follows from lemmas 3.3 and 2.9. If $p \in Z_{n}$, let $E \subset S_{k}$ be the $f$-exceptional curve and $F$ be the proper transform of the unique fibre of $\mathbb{F}_{n} \rightarrow \mathbb{P}^{1}$. Then $F$ is a (-1)-curve and its contraction gives a morphism $f^{\prime}: S=S_{3} \rightarrow \mathbb{F}_{n+1}$ which is the blow-up of a point $q \notin Z_{n+1} \subset \mathbb{F}_{n+1}$ and then the proof is as in the case $p \notin Z_{n}$. In particular, we have proven that for $k=3$, there is a morphism $f: S_{k} \rightarrow \mathbb{F}_{n}$ which is an isomorphism around $Z_{n}$, and there is an ample $\mathbb{Q}$-line bundle $L$ in $S_{k}$ such that $(S, L)$ is destabilised by the slope test configuration centred at the proper transform of $Z_{n}$.

Now, for the induction step, we suppose that we have an ample $\mathbb{Q}$-line bundle $L$ on $S_{k-1}$ and a composition of blow-ups $h: S_{k-1} \rightarrow \mathbb{F}_{n}$ such that $\left(S_{k-1}, L\right)$ is destabilised by the slope test configuration centred at the proper transform of $Z_{n}$ in $S_{k-1}$ and that $h$ is an isomorphism around $Z_{n}$. We let $f: S=S_{k} \rightarrow S_{2}=\mathbb{F}_{n}$ factor as $f=h \circ \pi$, where $h: S_{k-1} \rightarrow \mathbb{F}_{n}$ is as in the induction hypothesis and $\pi: S_{k} \rightarrow S_{k-1}$ is the blow-up at a point $p \in S_{k-1}$ with exceptional divisor $E \subset S_{k}$. Let $Z_{n}^{\prime}$ be the proper transform of $Z_{n}$ in $S_{k-1}$ via $h$. If $p \notin Z_{n}^{\prime}$, then the result follows from Lemma 2.9. 
Hence, suppose that $p \in Z_{n}^{\prime}$ and let $F_{k-1}$ (respectively $F_{k}$ ) be the proper transform in $S_{k-1}$ (respectively $S_{k}$ ) of the fibre $F$ of $\mathbb{F}_{n} \rightarrow \mathbb{P}^{1}$ passing through $h(p)$. Notice that $F_{k}^{2}=F_{k-1}^{2}-1$. Denote by $C_{i}\left(\right.$ respectively $\left.\widetilde{C}_{i}\right)$ the proper transform in $S_{k-1}$ (respectively $S_{k}$ ) of the exceptional divisor of $S_{i} \rightarrow S_{i-1}$ for $i=3, \ldots, k-1$. Let $F_{i}$ be the proper transform of $F$ in $S_{i}$ and let $l$ be the smallest index such that $F_{l}^{2}=-1$. Observe that if $l=k$ then $h$ is an isomorphism near $Z_{n}$ and $F$. By the induction hypothesis $\widetilde{C}_{i}^{2}=C_{i}^{2}$. The latter allows us to define the morphism $g: S_{k} \rightarrow S^{\prime}$ to a smooth surface $S^{\prime}$ as the successive contraction of $\widetilde{C}_{k}, \ldots, \widetilde{C}_{l+1}$, where we define $g$ to be the identity morphism if $k=l$. Then $\left(g\left(F_{k}\right)\right)^{2}=(g(E))^{2}=-1$. Let $\pi^{\prime}: S^{\prime} \rightarrow S^{\prime \prime}$ be the contraction of $g\left(F_{k}\right)$ to some smooth surface $S^{\prime \prime}$. Let $Z_{n}^{\prime \prime}=\left(\pi^{\prime} \circ g\right)\left(\widetilde{Z}_{n}\right) \subset S^{\prime \prime}$. Notice that $\left(\widetilde{Z}_{n}\right)^{2}=$ $\left(Z_{n}^{\prime \prime}\right)^{2}=-n-1$.

Hence, the composition $\pi^{\prime} \circ g$ is an isomorphism around $\widetilde{Z}_{n}^{\prime}$. Let $E^{\prime \prime}=\left(\pi^{\prime} \circ g\right)(E), \quad C_{l}^{\prime \prime}=\left(\pi^{\prime} \circ g\right)\left(\widetilde{C}_{l}\right), \ldots$, $C_{3}^{\prime \prime}=\left(\pi^{\prime} \circ g\right)\left(\widetilde{C}_{3}\right)$. By the inductive hypothesis $E \cdot C_{i}^{\prime \prime}=C_{i}^{\prime \prime} \cdot Z_{n}^{\prime \prime}=0$, for $i=3, \ldots, l$. Let $g^{\prime}: S^{\prime \prime} \rightarrow S^{\prime \prime \prime}$ be the successive contraction of $C_{l}^{\prime \prime}, \ldots, C_{3}^{\prime \prime}$. Hence $\left(g^{\prime}\left(Z_{n}^{\prime \prime}\right)\right)^{2}=\left(Z_{n}^{\prime \prime}\right)^{2}=-n-1$ and $\operatorname{rk}(\operatorname{Pic})\left(S^{\prime \prime \prime}\right)=2$. Hence $S^{\prime \prime \prime} \cong \mathbb{F}_{n+1}, g^{\prime}\left(Z_{n}^{\prime \prime}\right)=Z_{n+1}$ and $g^{\prime} \circ \pi^{\prime} \circ g: S_{k} \rightarrow \mathbb{F}_{n+1}$ is an isomorphism around $Z_{n+1}$, completing the proof of the inductive statement. In particular, given any rational surface $S$, we deduce that there is a morphism $S \rightarrow \mathbb{F}_{m}$ for some $m \geqslant 1$ such that $S$ is an isomorphism around $Z_{m}$, and an ample $\mathbb{Q}$-line bundle $L$ on $S$ such that, by means of Lemma 2.9, we construct a destabilising slope test configuration for $(S, L)$. The result for $S=\mathbb{P}^{1} \times \mathbb{P}^{1}$ and $S=\mathbb{P}^{2}$ follows from Lemma 3.2 and the fact that a pair $(S, L)$ admitting a cscK metric is K-polystable [6, 14].

Remark 3.5. If Conjecture 2.10 holds, we may expect a similar approach to answer Question 1.2 for other birational classes of surfaces as the one presented in the last proof. Namely, given a pair $(S, L)$, we may apply the Minimal Model Programme to find a morphism $S \rightarrow S_{0}$ where $S_{0}$ is a smooth surface with no (-1)-curves. Then we may classify all surfaces $S_{0}$ with no (-1)-curves such that $\operatorname{Amp}^{K}\left(S_{0}\right) \neq \operatorname{Amp}^{\mathbb{Q}}\left(S_{0}\right)$ and apply a solution to Conjecture 2.10. For all those surfaces with $\operatorname{Amp}^{K}\left(S_{0}\right)=\operatorname{Amp}^{\mathbb{Q}}\left(S_{0}\right)$, we may attempt to show that if enough (infinitely closed) points in $S_{0}$ are blown up, we may end-up with a morphism $g: S^{\prime} \rightarrow S_{0}$ such that $\operatorname{Amp}^{K}\left(S^{\prime}\right) \neq \operatorname{Amp}^{\mathbb{Q}}\left(S^{\prime}\right)$. Ideally, we should be able to describe the smallest such $S^{\prime}$ (in the case of rational surfaces $g$ is the identity, except for the special cases of $\mathbb{F}_{1}$ and $\mathbb{F}_{0}$ ). While a similar approach may also be considered in higher dimensions, a stronger statement than the one in Conjecture 2.10 would be needed to account for flips and flops.

\subsection{Some totally unstable rational surfaces}

The Matsushima-Lichnérowicz obstruction does not seem to have been fully explored in all obvious cases. There are two reasons for this: on the one hand it is not easy to describe the automorphism groups of varieties with many symmetries (i.e. those which are likely to have non-reductive automorphism groups), especially in higher dimensions. On the other hand, even when the birational class of a projective variety is well understood (e.g. rational surfaces), there may be many varieties in the class with very different automorphism groups depending on the choice of birational transformations among them. As a result, a complete classification of the cases for which Theorem 2.3 is applicable may be hopeless. Nonetheless we can conclude this article by proving Theorem 1.8, which can be stated in simple terms:

Proof of Theorem 1.8. The statement on $\mathbb{F}_{n}$ follows from Lemma 3.1 or Lemma 3.3. Consider (3.1). We are interested

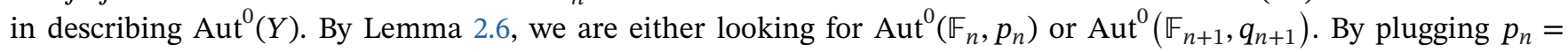
([x:0:0], [u:0]) with $x \neq 0, u \neq 0$ or $q_{n+1}=([0: 0: z],[0: v])$ with $z \neq 0, v \neq 0$ in (3.2) as fixed points, we get that the elements of $\operatorname{Aut}^{0}\left(\mathbb{F}_{n}, p_{n}\right) \cong \operatorname{Aut}^{0}(Y) \cong \operatorname{Aut}^{0}\left(\mathbb{F}_{n+1}, q_{n+1}\right)$ are of the form

$$
\left(a_{0} z_{0}^{n}+a_{1} z_{1} z_{0}^{n-1}+\cdots+a_{n} z_{1}^{n},\left(\begin{array}{ll}
a & b \\
0 & d
\end{array}\right) / \mu_{n}\right)
$$

where $a_{0}, \ldots, a_{n}, b \in \mathbb{C}, a, b \in \mathbb{C}^{*}$, computed by considering the stabiliser of $p_{n}$. Hence

$$
\operatorname{Aut}^{0}(Y) \cong\left(\mathbb{G}_{a}\right)^{n+1} \rtimes\left(\left(\mathbb{G}_{a} \rtimes \mathbb{G}_{m}^{2}\right) / \mu_{n}\right),
$$

which is not reductive by Lemma 2.5. The statement on the non-existence of cscK metrics follows from Theorem 2.3. Observe that if $\operatorname{Pic}(S) \leqslant 3$, then $S$ is a toric surface. Therefore, the non-existence of a cscK metric is equivalent, by the 
solution of the toric version of Conjecture 1.1 to toric-equivariant K-instability [17]. Hence there is an equivariant destabilising test configuration for $(S, L)$. But any equivariant test configuration is a test configuration in the sense of Definition 2.1 and hence $(S, L)$ is K-unstable.

\section{ACKNOWLEDGMENTS}

JMG was supported by the Simons Foundation under the Simons Collaboration on Special Holonomy in Geometry, Analysis and Physics (grant \#488631, Johannes Nordström). I would like to thank J. Blanc, D. Calderbank, I. Cheltsov, R. Dervan, J. Nordström, Y. Rubinstein and S. Sun for useful discussions. After the first version of this manuscript appeared on the Arxiv [22], and subsequently [12] introduced Calabi dream manifolds in their study of Conjecture 1.1, X. X. Chen was very kind to engage with me on discussions on the classification of Calabi dream manifolds. Furthermore, Y. Hashimoto explained to me how the initial proof of Theorem 1.3 did indeed classify strong Calabi dream rational surfaces. I thank them for those exchanges.

\section{ORCID}

Jesus Martinez-Garcia (D) https://orcid.org/0000-0002-0022-371X

\section{REFERENCES}

[1] V. Apostolov et al., Hamiltonian 2-forms in Kähler geometry. III. Extremal metrics and stability, Invent. Math. 173 (2008), no. 3, 547-601.

[2] V. Apostolov et al., Extremal Kähler metrics on projective bundles over a curve, Adv. Math. 227 (2011), no. 6, $2385-2424$.

[3] C. Arezzo and F. Pacard, Blowing up and desingularizing constant scalar curvature Kähler manifolds, Acta Math. 196 (2006), no. 2, 179-228.

[4] A. Beauville, Complex algebraic surfaces, London Math. Soc. Stud. Text, vol. 34, 2nd edn., Cambridge University Press, Cambridge, 1996, 10.1017/CBO9780511623936. Translated from the 1978 French original by R. Barlow, with assistance from N. I. Shepherd-Barron and M. Reid.

[5] R. J. Berman and B. Berndtsson, Convexity of the K-energy on the space of Kähler metrics and uniqueness of extremal metrics, J. Amer. Math. Soc. 30 (2017), no. 4, 1165-1196.

[6] R. J. Berman, T. Darvas, and C. H. Lu, Regularity of weak minimizers of the K-energy and applications to properness and K-stability, Ann. Sci. Éc. Norm. Supér. (4) 53 (2020), no. 2, 267-289.

[7] E. Calabi, The variation of Kähler metrics. i. the structure of the space; ii. a minimum problem, Bull. Amer. Math. Soc. 60 (1954), no. 2, 167-168.

[8] E. Calabi, Extremal Kähler metrics, Seminar on Differential Geometry, Ann. of Math. Stud., vol. 102, Princeton Univ. Press, Princeton, N.J., 1982. 259-290.

[9] I. Cheltsov and J. Martinez-Garcia, Stable polarized del Pezzo surfaces, Int. Math. Res. Not. IMRN 2020, no. 18, $5477-5505$.

[10] I. Cheltsov and J. Martinez-Garcia, Unstable polarized del Pezzo surfaces, Trans. Amer. Math. Soc. 372 (2019), no. 10, $7255-7296$.

[11] I. A. Cheltsov and Y. A. Rubinstein, On flops and canonical metrics, Ann. Sc. Norm. Super. Pisa Cl. Sci. (5) 18 (2018), no. 2, 1-29.

[12] X. Chen and J. Cheng, On the constant scalar curvature Kähler metrics, existence results, ArXiv e-prints (2018).

[13] X. X. Chen and G. Tian, Geometry of Kähler metrics and foliations by holomorphic discs, Publ. Math. Inst. Hautes Études Sci. (2008), no. 107, 1-107.

[14] T. Darvas and Y. A. Rubinstein, Tian's properness conjectures and Finsler geometry of the space of Kähler metrics, J. Amer. Math. Soc. 30 (2017), no. 2, 347-387.

[15] S. K. Donaldson, Scalar curvature and projective embeddings. I, J. Differential Geom. 59 (2001), no. 3, 479-522.

[16] S. K. Donaldson, Scalar curvature and stability of toric varieties, J. Differential Geom. 62 (2002), no. 2, $289-349$.

[17] S. K. Donaldson, Constant scalar curvature metrics on toric surfaces, Geom. Funct. Anal. 19 (2009), no. 1, 83-136.

[18] R. Hartshorne, Algebraic geometry, Grad. Texts Math, no. 52, Springer-Verlag, New York, 1977.

[19] C. LeBrun and S. R. Simanca, Extremal Kähler metrics and complex deformation theory, Geom. Funct. Anal. 4 (1994), no. 3, $298-336$.

[20] C. Li and C. Xu, Special test configuration and K-stability of Fano varieties, Ann. of Math. (2) 180 (2014), no. 1, $197-232$.

[21] A. Lichnérowicz, Sur les transformations analytiques des variétés kählériennes compactes, C. R. Acad. Sci., Paris 244 (1957), $3011-3013$. (French).

[22] J. Martinez-Garcia, Constant scalar curvature Kahler metrics on rational surfaces, ArXiv e-prints (2017).

[23] S. Mukai, An introduction to invariants and moduli, Cambridge Stud. Adv. Math., vol. 81, Cambridge University Press, Cambridge, 2003. Translated from the 1998 and 2000 Japanese editions by W. M. Oxbury.

[24] Y. Odaka, A generalization of the Ross-Thomas slope theory, Osaka J. Math. 50 (2013), no. 1, 171-185.

[25] J. Ross, Unstable products of smooth curves, Invent. Math. 165 (2006), no. 1, 153-162.

[26] J. Ross and R. Thomas, An obstruction to the existence of constant scalar curvature Kähler metrics, J. Differential Geom. 72 (2006), no. 3 , 429-466.

[27] Y. A. Rubinstein, Smooth and singular Kähler-Einstein metrics, Geometric and Spectral Analysis, Contemp. Math., vol. 630, Amer. Math. Soc., Providence, RI, 2014. 45-138.

[28] G. Székelyhidi, Extremal metrics and k-stability, Bull. Lond. Math. Soc. 39 (2007), no. 1, 76-84. 
[29] G. Tian, Kähler-Einstein metrics with positive scalar curvature, Invent. Math. 130 (1997), no. 1, 1-37.

[30] X. Wang, Height and GIT weight, Math. Res. Lett. 19 (2012), no. 4, 909-926.

How to cite this article: Martinez-Garcia J. Constant scalar curvature Kähler metrics on rational surfaces. Mathematische Nachrichten. 2021;1-12. https://doi.org/10.1002/mana.201900382 